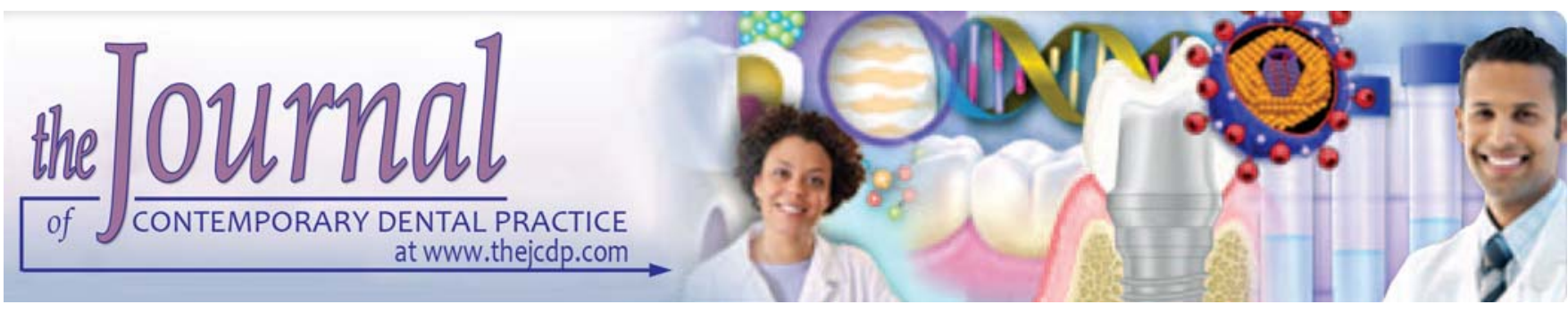

\title{
Sound Waves for Unsound Entities: An Electronic Search Study to Evaluate the Diagnostic Efficacy of Ultrasonography in Cysts and Tumors of Maxillofacial Region
}

DD Darshan, Girish Katti, GA Raviraj, NS Manikantan, AD Manoj Kumar, Dhanya Balakrishnan

\begin{abstract}
Objective: The purpose of this electronic search study was to evaluate the diagnostic efficacy of ultrasonography in cysts and tumors of maxillofacial region.
\end{abstract}

Materials and methods: An electronic search was performed for articles published from January 1989 to august 2009.

Results: Only 30 publications were searched and 22 publications showed high level of evidence (4 review articles, 12 clinical studies, 2 case reports, 1 quantitative analysis, 2 research articles and 1 preliminary report) with total of more than 2000 patients.

Conclusion: Ultrasonography is a noninvasive, low cost procedure, and recommended as a complimentary imaging modality.

Keywords: Ultrasonography (USG), Cyst, Tumor, USG guided needle biopsy.

How to cite this article: Darshan DD, Katti G, Raviraj GA, Manikantan NS, Kumar ADM, Balakrishnan D. Sound Waves for Unsound Entities: An Electronic Search Study to Evaluate the Diagnostic Efficacy of Ultrasonography in Cysts and Tumors of Maxillofacial Region. J Contemp Dent Pract 2013; 14(4):586-589.

Source of support: Nil

Conflict of interest: None declared

\section{INTRODUCTION}

The technology for producing ultrasound and the characteristics of sonic waves have been known for many years. Though the first attempt of practical application of ultrasound was to search the sunken titanic in 1912 and the medical application being used after World War II in late 1940s and early 1950s. The imaging techniques were not sufficiently developed those days. ${ }^{1}$

The recent advanced techniques in ultrasonography have proven that the ultrasound real time imaging is superior to ionizing radiation techniques in assessing cysts and tumors of maxillofacial region. ${ }^{2}$
As technology improved, CT has been used occasionally to aid in diagnosis of conditions in the bone. However, routine use of CT is associated with high radiation doses, Even though dose reduction methods have been established.

Low dose cone beam CT has been developed specifically for use in the dental and maxillofacial regions. MRI is another specialized imaging modality that does not involve the use of ionizing radiation and is important in imaging intracranial and soft tissue lesions. However, expensive equipment, increased scanning time, claustrophobia, contraindicated in patients with surgical clips, cardiac pacemakers and cochlear implants. Made the use of ultrasound imaging technology, a safe and minimally invasive procedure. ${ }^{2}$

The purpose of this electronic search study was to evaluate the diagnostic efficacy of ultrasonography in cysts and tumors of maxillofacial region.

\section{MATERIALS AND METHODS}

A literature search was conducted from January 1989 to August 2009.

The following keywords and Boolean operators were used:

'Ultrasonography (US) in oral cancer', 'US in oral malignancy', 'US in diagnosing oral malignancy', 'US in oral cysts', 'US in oral cysts and tumors', 'US in differentiating oral cysts and tumors', 'sonography in oral tumor metastasis', 'US in measuring tumor thickness', 'US in detecting tumor margins'.

The literature search was conducted in August 2009, using the following data base:

- PubMed: The national library of medicines online search interface for medline and premedline (http:// www.ncbi.nlm.nih.gov/entrez/query.fcgi)

- www.jiaomr.org 
- dmfr.birjournals.org

- Google India http://www.google.co.in

The primary focus or the search involved systematic reviews (evidence level Ia), experimental studies (evidence levels IIb) and case reports were considered. ${ }^{3}$

The levels of evidence of articles were classified according to the guidelines of the Oxford Center for Evidence-Based Medicine (http://www.cebm.net/levels_of_ evidence.asp). ${ }^{3}$

\section{DISCUSSION}

Ultrasonography (US) is a low-cost and widely available method with no radiation exposure. Ultrasound imaging is based on the phenomenon of reflection or refraction of sound waves at the interfaces between tissues of different acoustic properties. The beam received by the transducer is converted into electric current and displayed as an image or a graph.

Main indications for ultrasound in head and neck is to evaluate the swellings of the neck, particularly those involving the thyroid, cervical lymph nodes or the major salivary glands ultrasound is now regarded as the investigation of choice for detecting solid and cystic soft tissue masses, in detecting salivary gland and duct calculi, determination of the relationship of vascular structures and vascularitiy of masses with the addition of Doppler imaging, assessment of blood flow in the carotids and carotid body tumors. The advantage of ultrasound being sound waves are not ionizing radiation, there are no known harmful effects on any tissues at the energies and doses. Currently images show good differentiation between different soft tissues and are very sensitive for detecting focal disease in salivary glands. Techniques is widely available and relatively in expensive.

\section{ULTRASONOGRAPHY IN ODONTOGENIC CYSTS OF MAXILLOFACIAL REGION}

The study by Gundappa et al in 2007 has confirmed ultrasound as a reliable diagnostic technique for differentiating periapical cysts and granulomas, can provide accurate information on the pathological nature of the lesion, content of lesion, inflammatory process, capsular thickness of lesions. It can also differentiate between simple and complex cyst, and on nature of the content differentiate into solid and semi solid cysts. ${ }^{2,4}$

- Simple cysts appears totally anechoic lesions with limited or without internal echoes, e.g. radicular cyst

- Complex cysts appear with dense internal echoes or high echo, e.g. OKC

- Semisolid cysts when cystic and solid areas are combined in the same lesions
- Solid lesions have no liquid components and posterior enhancement and have a moderate echo.

By using color and power Doppler ultrasound allows to detected blood flow with in or around the lesion. ${ }^{2,4,5}$

\section{ULTRASONOGRAPHY IN SALIVARY GLAND TUMORS}

They are rare and approximately 1 to 3\% of all neoplasm's about $80 \%$ of salivary gland tumors arise from parotid gland, 60 to $80 \%$ of which are benign. In the small salivary gland, the ratio of benign to malignant tumors is higher.

When the patient is presented with a palpable mass in the salivary gland or mass is suspected, diagnostic ultrasonography can be employed to:

1. Confirm the presence of mass: Ultrasound can successfully detect any salivary gland mass. Detection of even small lesions was easy, especially in the parotid where its sensitivity approaches 100\%.

In a study which evaluated 302 salivary gland masses sonographically, gritzmann (1989) reported a $100 \%$ sensitivity in detection of salivary gland tumors. ${ }^{6-8}$

2. Differentiate between intra-and extraglandular lesions: Correct localization of masses is not a problem with US, and an accuracy of $98 \%$ has been reported. However, this might be difficult in the cervical part of the parotid gland (deep lobe). ${ }^{9,10}$

3. Suggests nature of the mass benign vs malignant: Identification of the mass is more difficult. Almost all of the salivary gland tumors (benign and malignant) have been reported as hypoechoic, thus an absolute differentiation is impossible.

Differentiation is possible to some extent based on additional findings for lesion being benign in nature, like the presence of sharp borders. Well-circumscribed tumors are more often benign. ${ }^{10}$

The nature of salivary gland tumors assessed by ultrasonography using the sharpness of the border as the sole indicator of benignity, on the contrary, a markedly heterogeneous structure and presence of fluid-filled cystic spaces (necrosis) frequently indicate a malignant tumor. Higher vascularity seen in color Doppler has also been suggested as an indicator for malignancy but in some occasions, malignant tumors demonstrate benign features. ${ }^{11,12}$

\section{ULTRASONOGRAPHY TO EVALUATE AND ASSESS THE TUMOR THICKNESS AND METASTASIS}

Malignant tumors of deep head and neck structures can invade skin, but the tumor periphery is difficult to assess clinically. High-resolution diagnostic ultrasound demonstrated the 
periphery of the tumor. The tumor can be distinguished from surrounding edema by its lower echogenicity and homogeneous echotexture, the maximum contour of the tumor can be marked with ink under ultrasound guidance which aids in excision and reconstruction planning. Ultrasound detection of close surgical margins had a sensitivity of $83 \%$ and a specificity of $63 \%$ and the tumor thickness measurement, showed a high degree of correlation with histology. ${ }^{11-14}$

\section{ULTRASONOGRAPHY IN ORAL CAVITY}

Diagnostic ultrasonography has been successfully applied in a number of areas in the oral cavity, predominantly for soft tissue assessment, generally tongue tumors and tumors of floor of the mouth appears to be hypoechoic in comparison to the surrounding muscles. ${ }^{15,16}$ Ultrasound applications in the palate have been reported to be successful for the determination of the location and the nature of tumors. ${ }^{17}$

\section{ULTRASONOGRAPHY IN NECK AND CERVICAL LYMPH NODES}

Over the past decade, high-resolution sonography has become a first line imaging modality for the evaluation of cervical space-occupying lesions including lymph nodes. ${ }^{18,19}$ In addition to the above, the contribution of contemporary color Doppler imaging in the diagnosis of vascular pathology and obstructions is remarkable. The thyroid gland, parathyroid gland and the major blood vessel tumors of the neck can be easily assessed. The high resolution ultrasonographic images currently available can detect neck nodes, reveal changes in the architecture of cervical lymph nodes, and assess their characteristics and the degree of vascular invasion. ${ }^{20-22}$

\section{ULTRASONOGRAPHY GUIDED NEEDLE BIOPSY IN CYSTS AND TUMORS OF MAXILLOFACIAL REGION}

Ultrasonography guided needle biopsy is a safe and efficient diagnostic procedure with an accuracy of $97 \%$ in the pathologic diagnosis of parotid masses. It can be performed in an outpatient clinic and enables specific tissue diagnosis to obviate intraoperative frozen biopsy and unnecessary surgery. An 18-gauge needle is sufficient for accurate and specific tissue diagnosis of parotid masses. ${ }^{23}$ Whereas the specificity in other parts of maxillofacial region is less when compared to salivary gland. ${ }^{24}$

\section{RESULTS}

Only 30 publications were searched and 22 publications showed high level of evidence (4 review articles, 12 clinical studies, 2 case reports, 1 quantitative analysis, 2 research articles and 1 preliminary report) with total of more than 2000 patients.

Hence ultrasound is a reliable diagnostic technique for differentiating periapical cysts and granulomas.

Ultrasound can detect any salivary gland mass with sensitivity being 100\%, differentiate between intra-and extra glandular lesions with an accuracy of $98 \%$ and can also differentiate benign and malignant lesion to some extent based on sharpness of the border and heterogeneity, it has a sensitivity of $83 \%$ and a specificity of $63 \%$ in detecting tumor thickness metastasis.

When applied to different tumors of oral cavity it can successfuly determinate the location and the nature of tumors.

The high resolution ultrasonographic images currently available can detect neck nodes, reveal changes in the architecture of cervical lymph nodes, and assess their characteristics and the degree of vascular invasion and currently can also guide in biopsies of different maxillofacial pathology.

\section{CONCLUSION}

Few studies and case reports showed high level of evidence and showed ultrasound real-time imaging is a technique that may help make a differential diagnosis between cysts but not establish definitive diagnosis, it revealed the nature of the content of a lesion. The additional use of color and power Doppler ultrasound allows to detected blood flow with in or around the lesion. In regard to the head and neck tumors, there has been a great deal of interest in the imaging of salivary gland, floor of mouth, tongue and palatal tumors.

Several researchers have studied the ultrasonographic features in conforming the tumor mass, metastasis, lymph nodal involvement, tumor thickness and in differentiating benign and malignant lesion for some extent, in an attempt to make a diagnosis before biopsy as the surgical management of these tumors may vary. Also ultrasonography guided needle biopsy is a safe and efficient diagnostic procedure.

It is quite difficult to compare or even combine their outcomes in a scientifically meaningful manner. Because of insufficient research data, there is a need for high-quality randomized, controlled trials in this area of medicine. Finally ultrasonography is a noninvasive, low cost procedure, and recommended as a complimentary imaging modality.

\section{REFERENCES}

1. Curry TS, James E, Dowdy, Marry RC. Christensens physics of diagnostic radiology. 4th ed. Philadelphia 1990.

2. Gundappa M, Ng SY, Whaites EJ. Comparison of ultrasound, digital and conventional radiography in differentiating periapical lesions. Dentomaxillofacial Radiology 2006;35:326-333. 
3. Phillips B, Ball C, Sackett D, Badenoch D, Straus S, Haynes B, Dawes M. Oxford Centre for Evidence-based Medicine-Levels of Evidence. Since November 1998. Updated by Jeremy Howick March 2009.

4. Cotti E, Campisi G, Ambu R, Dettori C. Ultrasound real time imaging in the differential diagnoses of periapical lesions. Int Endod J 2003 Aug;36(8):556-563.

5. Raczkowska-Siostrzonek A, Koszowski R, Morawiec T. Ultrasonography in detection of periapical lesions-preliminary report. Dent Med Probl 2007;44(4):445-448.

6. Gritzmann N, Rettenbacher T, Hollerweger A, et al. Sonography of the salivary glands. Eur Radiol 2003;13:964-975.

7. Gritzmann N. Sonography of the salivary glands. Am J Roentgenol 1989;153:161-166.

8. Biaek EJ, Jakubowski W, Karpiska G. Role of ultrasonography in diagnosis and differentiation of pleomorphic adenoma. Arch Otolaryngol Head Neck Surg 2003;129:929-933.

9. Kim J, Kim EK, Park CS, Choi YS, Kim YH, Choi EC. Characteristic sonographic findings of warthins tumor in the parotid gland. Journal of Clinical Ultrasound 32(2):78-81.

10. Yasumoto M, Shibuya H, Gomi N, Kasuga T. Ultrasonographic appearance of dermoid and epidermoid cysts in the head and neck. J Clinical Ultrasound. Oct 1991;19(8):455-461.

11. Songra AK, Ng SY, Farthing P, Hutchison IL, Bradley PF. Observation of tumour thickness and resection margin at surgical excision of primary oral squamous cell carcinoma-assessment by ultrasound 2005. Int J Oral Maxillofac Surg 2006 April; 35(4):324-331.

12. Natori T, et al. Usefulness of intraoral ultrasonography to predict neck metastasis in patients with tongue carcinoma. Oral Diseases 2007;14(7):591-599.

13. Shaik S, Panda N, Singh SB, Katariya S, Kalagara S. The role of ultrasonography in the management of tumors of the neck. Ear, Nose and Throat Journal Aug 1, 2000.

14. Adoga AA, Nimkur TL, Manasseh AN, Echejoh GO. Buccal soft tissue lipoma in an adult Nigerian: a case report and literature review. Journal of Medical Case Reports 2008;2:382.

15. Ishii J, Nagasawa $H$, Wadamori $T$, Yamashiro $M$, Ishikawa $H$, Yamada T, Miyakura T, Amagasa T. Ultrasonography in the diagnosis of palatal tumors. Oral Surg Oral Med Oral Path Oral Radiol Endodontics 1999 Jan;87(1):39-43.

16. Kaneoya A, Hasegawa S, Tanaka Y, Omura K. Quantitative analysis of invasive front in tongue cancer using Ultrasonography J Oral and Maxillofac Surg 67(1):40-46.

17. Shintani S, et al. The usefulness of intraoral ultrasonography in the evaluation of oral cancer. Int J Oral Maxillofac Surg 2001; 30(2):139-143.

18. Shaw L, Al-Malt A, Carlan SJ, Plumley D, Greenbaum L, Kosko J. Three-dimensional ultrasonographic findings and clinical implications. J Ultrasound Med 2004;23:1121-1124.
19. Wan YL, Chan SC, Chen YL, Cheung YC, Lui KW, Wong HF, Hsueh C, See LC. Ultrasonography-guided core needle biopsy of parotid gland masses. American Society of Neuroradiology 2004 Oct;25:1608-1612.

20. Lin JD, Hsueh C, Liu FH, Chen JY, Chang HY. Clinical application of thyroid ultrasonography, the endocrinologist 2006;16(6):319-322.

21. Bui A, Mazzaferri EL. New paradigms in the diagnosis and management of thyroid nodules. The Endocrinologist 2007; 17(1):35-45.

22. Sumer AP, Danaci M, Sandikei EO, Sumer M, Celenk P. Ultrasonography and Doppler ultrasonography in the evaluation of intraosseous lesions of the jaws. Dentomaxillofacial Radiology 2009;38:23-27.

23. Howlett DC. Diagnosing a parotid lump: fine needle aspiration cytology or core biopsy? Br J Radiol 2006;79:295-297.

24. Ng SY, Songra AS, Hutchison LL. Noval use of ultrasound guided surface marking of head and neck tumors invading facial skin. Oral Surg Oral Med Oral Path Oral Radiol Endodontics 2006 April;101(4):499-504.

\section{ABOUT THE AUTHORS}

\section{DD Darshan (Corresponding Author)}

Assistant Professor, Department of Oral Medicine and Radiology KVG Dental College and Hospital, Sullia, Karnataka, India, Phone: 9986220538, e-mail: darshandevang@gmail.com

\section{Girish Katti}

Professor and Head, Department of Oral Medicine and Radiology Al-Badar Dental College and Hospital, Gulbarga, Karnataka, India

\section{GA Raviraj}

Reader, Department of Pedodontics, KVG Dental College and Hospital, Sullia, Karnataka, India

\section{NS Manikantan}

Reader, Department of Prosthodontics, KVG Dental College and Hospital, Sullia, Karnataka, India

\section{AD Manoj Kumar}

Reader, Department of Prosthodontics, KVG Dental College and Hospital, Sullia, Karnataka, India

\section{Dhanya Balakrishnan}

Reader, Department of Conservative Dentistry and Endodontics, KVG Dental College and Hospital, Sullia, Karnataka, India 